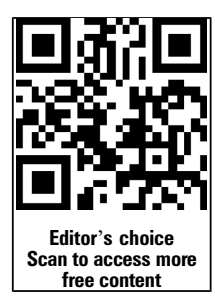

${ }^{1}$ Department of Clinical Pathology, Faculty of Medicine, Health Sciences Center, Kuwait University, Kuwait, Kuwait ${ }^{2}$ Department of Anatomical Pathology, Toronto General Hospital, University Health Network, Toronto, Ontario, Canada

\section{Correspondence to} Dr Rola H Ali, Department of Clinical Pathology, Faculty of Medicine, Health Sciences Center, Kuwait University, PO Box 24923 Safat, Kuwait 13110, Kuwait; rolapathology@gmail.com

Received 14 December 2014 Accepted 20 December 2014 Published Online First 16 January 2015

\title{
Endometrial stromal tumours revisited: an update based on the 2014 WHO classification
}

\author{
Rola H Ali, ${ }^{1}$ Marjan Rouzbahman ${ }^{2}$
}

ABSTRACT

Endometrial stromal tumours (EST) are rare tumours of endometrial stromal origin that account for less than $2 \%$ of all uterine tumours. Recent cytogenetic and molecular advances in this area have improved our understanding of ESTs and helped refine their classification into more meaningful categories. Accordingly, the newly released 2014 WHO classification system recognises four categories: endometrial stromal nodule (ESN), low-grade endometrial stromal sarcoma (LGESS), high-grade endometrial stromal sarcoma (HGESS) and undifferentiated uterine sarcoma (UUS). At the molecular level, these tumours may demonstrate a relatively simple karyotype with a defining chromosomal rearrangement (as in the majority of ESNs, LGESSs and YWHAE-rearranged HGESS) or demonstrate complex cytogenetic aberrations lacking specific rearrangements (as in UUSs). Herein we provide an update on this topic aimed at the practicing pathologist.

\section{INTRODUCTION}

Endometrial stromal tumours (EST) are uncommon uterine tumours responsible for less than $10 \%$ of uterine mesenchymal neoplasms ${ }^{1}$ and less than $2 \%$ of all uterine neoplasms. The diagnostic criteria and nomenclature of ESTs have gone through several modifications in the past four decades since the earliest study by Norris and Taylor. ${ }^{2}$ The rarity of these tumours coupled with the overlapping morphological features have contributed to the difficulty in classifying ESTs into clinically meaningful categories.

Briefly, endometrial stromal sarcomas (ESS) were originally divided into low-grade and high-grade based on mitotic count, ${ }^{2}$ however, further studies have demonstrated that mitotic activity is prognostically irrelevant. Later on, the 2003 WHO classification system eliminated the 'high-grade' category and reclassified these tumours into 'ESS' (low-grade tumours with histological resemblance to proliferative endometrial stroma) and 'undifferentiated endometrial sarcoma' (pleomorphic tumours with no resemblance to endometrial stroma). Subsequently, it has become increasingly evident that the 'undifferentiated endometrial sarcoma' category is too broad, encompassing a wide range of heterogeneous tumours with different clinical behaviour and outcome, and that at least a subset of these tumours are morphologically and genetically distinct. The 2014 WHO classification scheme now incorporates recent molecular findings into the classification, dividing ESTs into endometrial stromal nodule (ESN), low-grade endometrial stromal sarcoma (LGESS), high-grade endometrial stromal sarcoma (HGESS) and undifferentiated uterine sarcoma (UUS).

\section{ENDOMETRIAL STROMAL NODULE \\ Overview}

ESNs are rare benign tumours of the uterus. The two largest series published to date showed no recurrences after a follow-up period of up to 16 years and 17.8 years, respectively. ${ }^{3}{ }^{4}$ ESN occurs over a wide age range, from 31 to 86 years with a mean of 53 years in one study, ${ }^{4}$ usually presenting with abnormal vaginal bleeding or as an incidental finding in hysterectomy specimens performed for other reasons.

\section{Morphological features}

Grossly, ESN is a well-circumscribed tumour of variable size that may occur as an intramural mass or as a polypoid tumour protruding into the endometrial cavity. It usually has a fleshy and soft yellow to tan cut surface that may contain cystic areas. Examples with firm white cut surface resembling a leiomyoma usually show extensive stromal fibrosis histologically.

Microscopically, ESN is expansile in nature lacking myometrial invasion, yet focal finger-like projections into the adjacent myometrium (less than 3 in number and less than $3 \mathrm{~mm}$ in length) are accepted. ${ }^{3}$ Lymphovascular invasion must be absent. Tumours with clear-cut myometrial/lymphovascular invasion should be diagnosed as LGESS instead. ESNs are classically densely cellular, although occasional hypocellular examples with fibrous or fibromyxoid differentiation are encountered, ${ }^{4}$ and are characterised by whorling around spiral arteriole-like vessels. The tumour cells resemble those of proliferative endometrial stroma, exhibiting minimal cytological atypia. Mitotic activity is usually low (up to 5 per 10 high power fields) but a higher count does not exclude the diagnosis. ${ }^{4}$ ESNs demonstrate diverse morphological variations, which include smooth muscle, skeletal muscle and sex-cord stromal differentiation ${ }^{5-8}$ as well as a variety of other secondary features such as hyalinisation, collagen plaques, foamy macrophages and cholesterol clefts.

A definitive diagnosis of ESN can only be rendered after careful sampling and examination of the tumour border, which is only possible on hysterectomy specimens. On curettage specimens, the identification of aglandular stromal fragments $\geq 5 \mathrm{~mm}$ in size should raise suspicion of an underlying EST of some sort, ${ }^{9}$ with a caveat that the distinction between ESN and LGESS may be impossible in such specimens. Occasional cases, so-called 'EST with limited infiltration', show more tumour border irregularities than is usually expected in conventional ESNs, yet the infiltration is not as extensive as that of LGESS. ${ }^{4}$ In the
Rouzbahman M. J Clin

Pathol 2015;68:325-332. 
current WHO classification, this is not recognised as a distinct diagnostic category, but the available limited clinical data suggest a benign course.

\section{Ancillary studies and differential diagnosis}

ESN and LGESS share the same immunohistochemical profile (discussed below). As in LGESS, $\mathrm{t}(7 ; 17)$ resulting in JAZF1SUZ12 gene fusion is the most common chromosomal rearrangement, identified in over half of the cases, conventional as well as variant ESNs. ${ }^{10}$

The most important differential diagnoses are LGESS and cellular/highly cellular leiomyoma (table 1). ${ }^{11}$ Distinction from LGESS basically relies on exclusion of myometrial and lymphovascular invasion, as the morphology is indistinguishable. Smooth muscle differentiation in ESN can be particularly confusing as one may misinterpret the interdigitating metaplastic smooth muscle as myometrial invasion. Delineating the true tumour border at gross examination is extremely helpful in this case. Highly cellular leiomyomas contain large thick-walled blood vessels, cleft-like spaces and at least focal conventional fascicular pattern. ${ }^{11}$ Although smooth muscle tumours and ESTs have an overlapping immunophenotype, an immunohistochemical panel of CD10 and at least two muscle markers (such as desmin, h-caldesmon, smooth muscle heavy chain myosin and HDAC8) usually helps in this distinction. ${ }^{12-15}$

\section{LOW-GRADE ENDOMETRIAL STROMAL SARCOMA \\ Overview}

LGESS is the second most common malignant mesenchymal tumour of the uterus, ${ }^{16}$ and it is much more common than ESN. The age range is somewhat similar to that of ESN, with the majority occurring in the perimenopausal period. It occasionally arises in young women and adolescents. ${ }^{17-20}$ Patients usually present with vaginal bleeding or pelvic pain. The uterine corpus is the most frequent location though it can also primarily arise in a variety of extrauterine locations such as ovary, ${ }^{21}$ pelvis, abdominal cavity, vulva and vagina. It often occurs in association with endometriosis. ${ }^{22-27}$

LGESS is a slow-growing tumour with an indolent clinical course characterised by multiple and/or late relapses, some occurring as late as 20 years after hysterectomy. ${ }^{28}$ The relapses may be local (pelvic/vaginal), distant (most common are abdominal

Table 1 Differential diagnoses of endometrial stromal tumours

\begin{tabular}{llll}
\hline ESN & LGESS & HGESS & UES \\
\hline $\begin{array}{llll}\text { Cellular } \\
\text { leiomyoma }\end{array}$ & $\begin{array}{l}\text { Gland-poor } \\
\text { adenomyosis }\end{array}$ & LGESS & Leiomyosarcoma \\
LGESS & $\begin{array}{l}\text { Cellular leiomyoma } \\
\text { Intravascular }\end{array}$ & Leiomyosarcoma & HGESS \\
& leiomyomatosis & UUS & $\begin{array}{l}\text { Undifferentiated } \\
\text { carcinoma }\end{array}$ \\
& $\begin{array}{l}\text { Leiomyosarcoma with } \\
\text { extensive intravascular }\end{array}$ & GIST & $\begin{array}{l}\text { Carcinosarcoma } \\
\text { component }\end{array}$ \\
& HGESS & & \\
& & & $\begin{array}{l}\text { Adenosarcoma with } \\
\text { stromal overgrowth } \\
\end{array}$ \\
& UTROSCT & & Lymphoma \\
& PEComa & & Melanoma \\
& GIST & & \\
\hline
\end{tabular}

ESN, endometrial stromal nodule; GIST, gastrointestinal stromal tumour; HGESS, high-grade endometrial stromal tumour; LGESS, low-grade endometrial stromal tumour; PEComa, perivascular epithelioid cell tumour; UTROSCT, uterine tumour resembling ovarian sex-cord tumour; UUS, undifferentiated uterine sarcoma. cavity/wall and lungs), or both (figure 1). ${ }^{29}$ Relapse rates up to $56 \%$ have been reported in patients with early-stage disease possibly due to inadvertent tumour morcellation at surgery. ${ }^{30} 31$ The stage is the most important prognostic factor. ${ }^{32}$

Currently, total hysterectomy and bilateral salpingooophorectomy, with or without adjuvant therapy, are the mainstay of treatment for stage I-II LGESS. ${ }^{33}{ }^{34}$ Lymph node metastasis can occur, ${ }^{35}$ however, lymphadenectomy is unlikely to improve survival. ${ }^{36-38}$ Reports on conservative fertility-sparing management in young patients are emerging though experience with this approach is still limited. ${ }^{17} 202939$ Advanced-stage (III-IV) disease is managed with cytoreductive surgery and adjuvant hormonal therapy usually in the form of progestins or aromatase inhibitors. ${ }^{3140}$ Radiation therapy may be useful for local control but is not universally recommended. ${ }^{37} 4142$ Tamoxifen and oestrogen replacement therapy are contraindicated as they promote disease progression. ${ }^{43}$

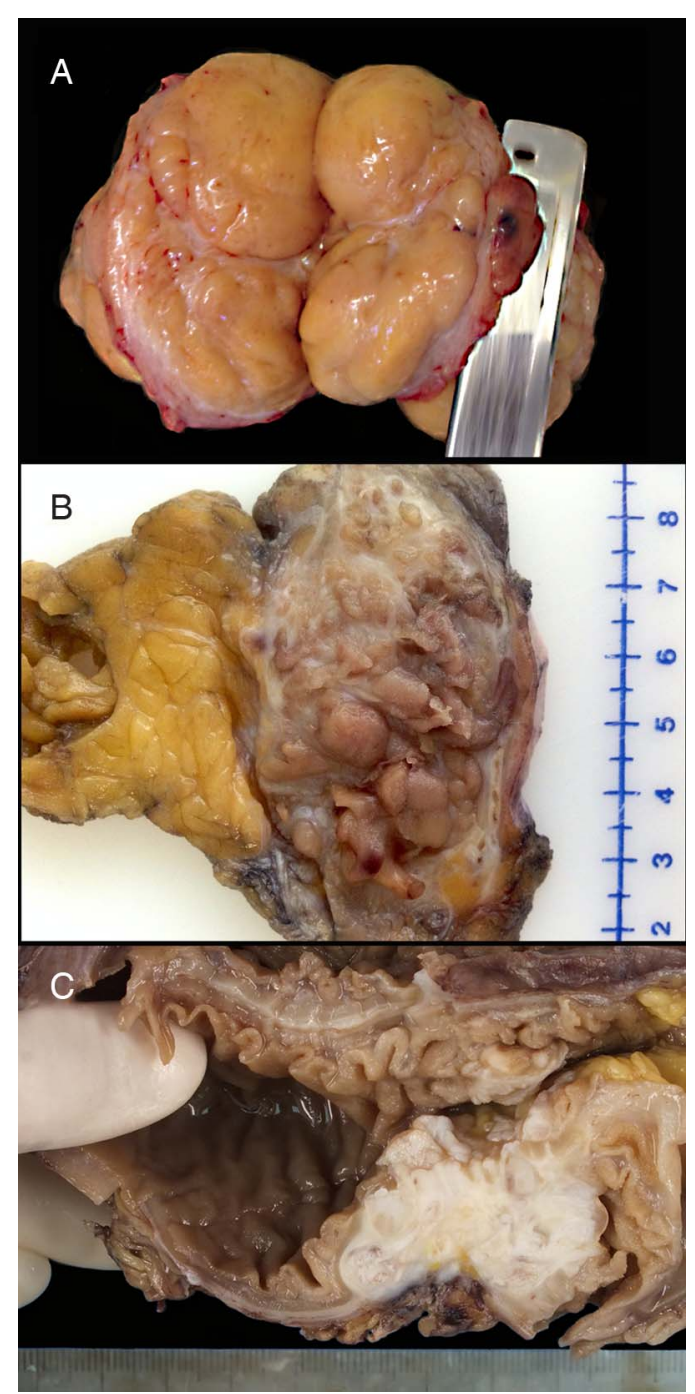

Figure 1 Gross appearance of low-grade endometrial stromal sarcoma (LGESS). (A) A 'myomectomy' specimen showing soft yellow cut surface, proved to be LGESS histologically. Courtesy of Dr Remaa Al-Safi, Department of Pathology, Maternity Hospital, Kuwait.

(B) Recurrent LGESS in the soft tissues of abdominal wall following hysterectomy, showing bulging 'wormlike' masses of tissue reminiscent of that seen in the uterus. (C) Recurrent LGESS in the abdominal cavity involving intestinal wall showing white rubbery appearance corresponding to extensive hyaline plaques. 


\section{Morphological features}

Grossly, LGESS may be submucosal or intramural, usually with ill-defined borders and 'wormlike' permeation within the myometrium and parametrial tissue, though some tumours might appear relatively circumscribed. Similar to ESN, the cut surface is fleshy tan to yellow (figure $1 \mathrm{~A}, \mathrm{~B}$ ), but may occasionally be firm white when there is extensive fibrous stroma (figure 1C). Areas of haemorrhage, necrosis and cystic degeneration might be present. ${ }^{44}$ Infrequently, LGESS appears as a pure cystic mass.

The 'tongue-like' patterns of myometrial and lymphovascular invasion are classical histological features that are important in the distinction from ESN (figure 2A, E). Similar to ESN though, LGESS is composed of sheets of small uniform cells with oval to spindle nuclei, whorling around spiral arteriole-like vessels (figure $2 \mathrm{~F})$. The neoplastic cells resemble those of proliferative endometrial stroma and lack significant cytological atypia. ${ }^{25}$ Mitotic activity is variable. Variant histological features include smooth muscle differentiation ('star-burst' appearance), rhabdomyoblastic, rhabdoid, fibromyxoid, sex-cord, glandular, collagen plaques and others (figure $2 \mathrm{~B}-\mathrm{D}$ ). ${ }^{46-51}$ As discussed above, it is not possible to separate LGESS from ESN on curettage specimens (figure $2 \mathrm{G}$ ).

\section{Ancillary studies and differential diagnosis}

LGESS is positive for CD10 (figure $2 \mathrm{H}$ ), oestrogen receptor (ER) and progesterone receptor (PgR). However, CD10 is not entirely specific and may stain some smooth muscle tumours, especially leiomyosarcoma and almost $50 \%$ of highly cellular leiomyomas. Conversely, false-negative CD10 staining can be encountered with underfixation. Therefore, it is important to use a panel of immunostains that includes CD10 and at least two smooth muscle markers (eg, desmin, h-caldesmon, smooth muscle heavy chain myosin, HDAC8) as there is no single marker that is specific for ESS. ${ }^{52}$ Not uncommonly, LGESSs stain with smooth muscle markers, especially at foci of muscle differentiation but also in conventional areas (depending on the marker used). Adding h-Caldesmon is often helpful as it has good sensitivity and specificity for smooth muscle differentiation. ${ }^{53}$ So it is important that one takes into account the relative intensity and distribution of these stains and tries to interpret them in view of the histological features, gross findings and clinical picture. Foci of sex-cord differentiation stain with calretinin, inhibin, WT1, Melan-A and CD99.

Important differential diagnoses for uterine LGESS include smooth muscle tumours (intravascular leiomyomatosis, cellular and highly cellular leiomyoma, leiomyosarcoma), adenomyosis with sparse glands, perivascular epithelioid cell tumour (PEComa) and uterine tumour resembling ovarian sex cord tumour (UTROSCT; table 1). Attention to histological features and use of appropriate immunostains are often helpful. For example, LGESS with myxoid change (figure 2C) can be

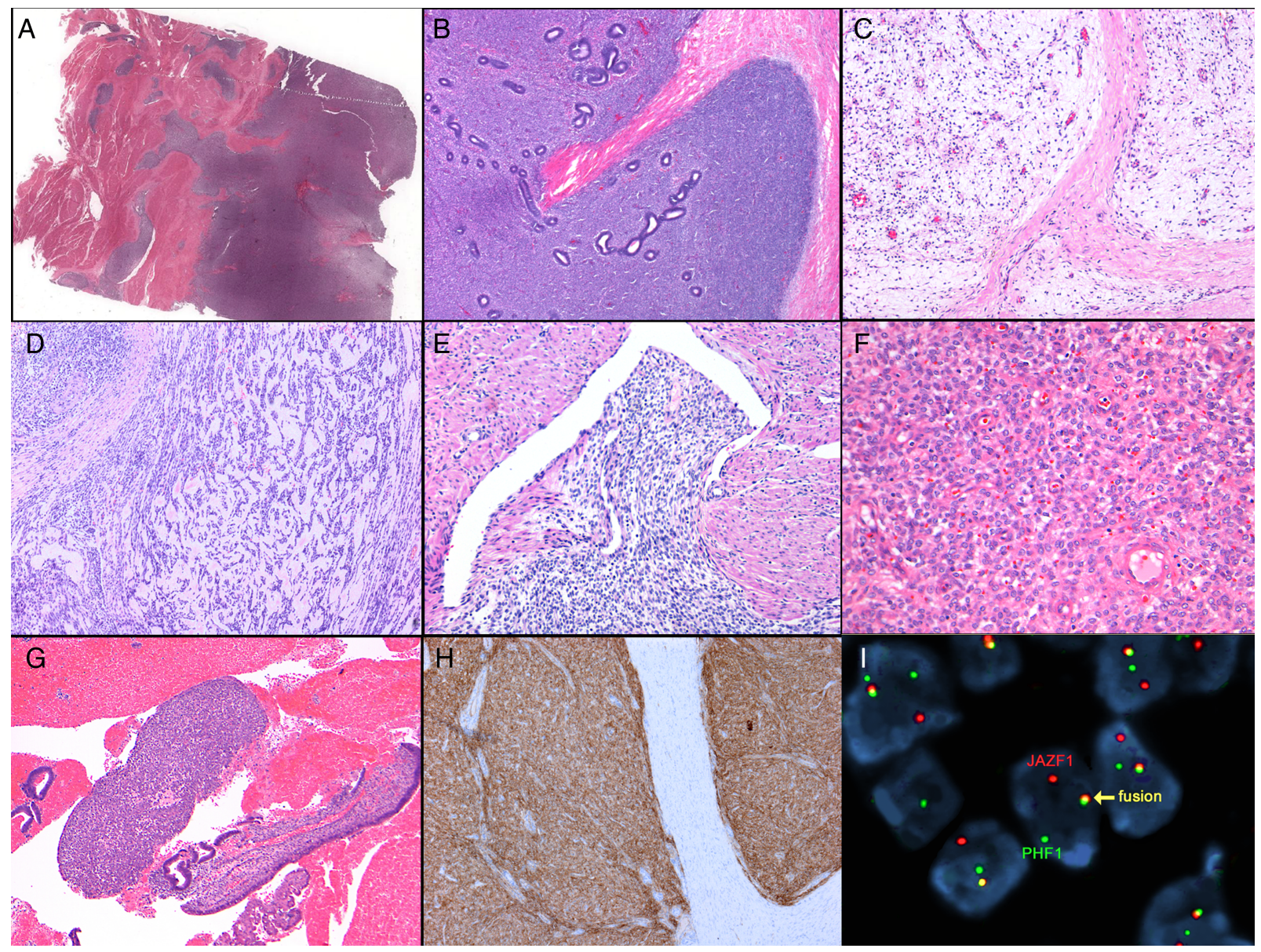

Figure 2 Microscopic appearance of low-grade endometrial stromal sarcoma (LGESS). (A) JAZF1-rearranged LGESS at scanning magnification with 'tongue-like' infiltration. (B) Endometrioid glandular elements are rarely seen (H\&E $\times 5)$. (C) PHF1-rearranged LGESS with striking myxoid change and preservation of arteriolar pattern (H\&E $\times 10)$. (D) Extensive hyaline plaques (H\&E $\times 5)$. (E) Lymphovascular invasion $(H \& E \times 10)$. (F) Spiral arteriole-like vessels and sprinkle of 'stromal granulocytes' are helpful features (H\&E $\times 10)$. (G) Curettage specimen showing fragments of aglandular endometrial stroma, which proved to be LGESS on hysterectomy $(H \& E \times 10)$. (H) CD10 immunostain positivity $(C D 10 \times 5)$. (I) Fluorescence in situ hybridisation (FISH) fusion assay showing JAZF1-PHF1 fusion (yellow signal). 
confused with myxoid smooth muscle lesions, but the 'tonguelike' infiltration, small arterioles, presence of classic areas of LGESS elsewhere, along with the CD10 immunostaining and muscle marker negativity, will usually resolve the diagnosis. ${ }^{54}$

The differential diagnosis for extrauterine LGESS depends on the location of the tumour. In case of ovarian location, metastasis from a primary uterine LGESS and ovarian sex cord-stromal tumours should be excluded. ${ }^{21}$ In the abdominal cavity, gastrointestinal stromal tumour (GIST) comes into the differential and should be excluded with the combined use of cKit and DOG1 immunostains.

The majority of LGESSs, including conventional and variant tumours, harbour chromosomal rearrangements, ${ }^{10} 18 \quad 55-58$ but at present there seems to be no correlation between the histological variant and the underlying genotype. The most common rearrangement, $\mathrm{t}(7 ; 17)(\mathrm{p} 15 ; \mathrm{q} 21)$, results in JAZF1-SUZ12 gene fusion. ${ }^{59}$ Other gene fusions include JAZF1-PHF1 (figure 2I), and the much less common EPC1-PHF1, MEAF6-PHF1, ${ }^{60} 61$ ZC3H7-BCOR ${ }^{62}$ and MBTD1-CXorf67. ${ }^{63}$ Molecular testing, however, is not routinely performed but may be helpful when dealing with cases of unusual location or morphology.

\section{HIGH-GRADE ENDOMETRIAL STROMAL SARCOMA Overview}

One of the main highlights of the updated 2014 WHO classification of uterine mesenchymal tumours ${ }^{16}$ is the re-introduction of HGESS as a distinct entity. The identification of YWHAE$N U T M 2 A / B$ (also known as YWHAE-FAM22A/B) gene fusion ${ }^{64}$ as a recurrent event in this more aggressive subset of tumours has provided objective support to the existence of a subcategory of ESS that is intermediate between LGESS and UUS. Currently, the term HGESS is reserved for those rare stromal sarcomas exhibiting round cell morphology and characteristic immunophenotype, and harbouring a $t(10 ; 17)(\mathrm{q} 22 ; \mathrm{p} 13)$ rearrangement with YWHAE$N U T M 2 A / B$ gene fusion. In the past, at least some of these tumours may have been lumped under 'undifferentiated endometrial sarcoma'. The paper by Kurihara et al ${ }^{65}$ has been instrumental in drawing attention to the heterogeneity of the undifferentiated tumour category, stressing the importance of separating tumours within this category into those with nuclear uniformity (some may have been YWHAE-rearranged) from those with nuclear pleomorphism (real undifferentiated sarcomas characterised by complex karyotypes and frequent p53 alterations). Additionally, separating HGESS from LGESS is as important since they appear to be more aggressive clinically with frequent extrauterine disease at initial presentation. ${ }^{66}{ }^{67}$ Based on the limited data available, the prognosis of YWHAE-rearranged cases seems to be intermediate between that of LGESS and UUS. ${ }^{66} 68$ These tumours usually present as abnormal vaginal bleeding similar to LGESS. Table 2 summarises the molecularly confirmed HGESS cases reported to date.

\section{Morphological features}

On gross examination, HGESS may be polypoid and usually shows extensive permeative growth through the uterine wall akin to LGESS, frequently with haemorrhage and necrosis. ${ }^{66}$ Microscopically, HGESS demonstrates the 'tongue-like' permeative pattern into the myometrium and angiolymphatic invasion typical of LGESS (figure 3A). The cytomorphology, however, is distinctive and does not exactly look like proliferative endometrial stromal cells. It is characterised by a monomorphic proliferation of round cells in a vaguely nested or pseudoglandular pattern $^{66}$ (figure 3B-D). About half of the tumours are biphasic, where the round-cell component is admixed with an LGESS-like component that is usually fibrous or fibromyxoid. ${ }^{66}$ The round cells are larger than those of LGESS with slightly irregular nuclear contour, fine evenly dispersed chromatin with nuclear clearing and lack of prominent nucleoli, while the cytoplasm may be scant to moderate in amount, imparting an epithelioid appearance (figure 3D). The tumour displays delicate curvilinear vasculature that is different from the spiral arterioles seen in LGESS (figure 3C). Mitotic activity is increased and necrosis is common. While YWHAE-rearranged endometrial sarcomas consistently show uniform high-grade cytomorphology, the reverse is not always true (ie, not all uniform high-grade endometrial sarcomas are YWHAE-associated). Sciallis et $a l^{69}$ recently identified three morphological patterns of non-pleomorphic HGESSs, and found that the YWHAE rearrangement was only detected in a subgroup displaying the characteristic morphological features described above along with the characteristic immunophenotype (below).

Table 2 Molecularly confirmed high-grade endometrial stromal sarcoma (HGESS) cases in the literature

\begin{tabular}{|c|c|c|c|c|c|}
\hline Author & Year & Number of cases & Molecular method & $\begin{array}{l}\text { FIGO stage at } \\
\text { presentation }\end{array}$ & $\begin{array}{l}\text { Presence of LGESS-like } \\
\text { component histologically* }\end{array}$ \\
\hline Ali et al ${ }^{18}$ & 2014 & 1 & FISH breakapart & IIIc & $0 / 1$ \\
\hline Gremel et a $\left.\right|^{68}$ & 2014 & 3 & RT-PCR & NA & NA \\
\hline Sciallis et al ${ }^{69}$ & 2014 & 4 & FISH breakapart & $\begin{array}{l}1 / 4 \text { stage IIIB } \\
1 / 4 \text { stage IIIC } \\
2 / 4 \text { stage IVB }\end{array}$ & NA \\
\hline Stewart et $\left.a\right|^{55}$ & 2014 & 4 & FISH breakapart & NA & $1 / 4$ \\
\hline Croce et $a l^{70}$ & 2013 & 6 & FISH breakapart and RT-PCR & $\begin{array}{l}3 / 6 \text { stage IB } \\
1 / 6 \text { stage IVB } \\
2 / 6 \mathrm{NA}\end{array}$ & $1 / 6$ at least $\dagger$ \\
\hline Lee et $a l^{66} \ddagger$ & 2012 & 13 & FISH breakapart and karyotyping & $\begin{array}{l}10 / 12 \text { stages II to III } \\
2 / 12 \text { stage I }\end{array}$ & $8 / 13$ \\
\hline Amant et $a l^{71}$ & 2011 & 1 & $\mathrm{t}(10 ; 17)$ by karyotyping and M-FISH & IIIc & NA \\
\hline Regauer et $a l^{72}$ & 2008 & 1 & $\mathrm{t}(10 ; 17)$ by karyotyping and M-FISH & Recurrent abdominal mass & $1 / 1$ \\
\hline Micci et $a l^{73}$ & 2003 & 1 & $\mathrm{t}(10 ; 17)$ by karyotyping and RxFISH & Metastatic to lung & NA \\
\hline Leunen et $a l^{74}$ & 2003 & 1 & $\mathrm{t}(10 ; 17)$ by karyotyping & III & NA \\
\hline
\end{tabular}

*Some YWHAE-rearranged HGESSs contain an LGESS-like component that is fibrous or fibromyxoid.

tThree additional cases were described as 'spindle' with no details on the grade of these spindle areas.

fOriginal paper that described YWHAE rearrangement in HGESS.

LGESS, low-grade endometrial stromal sarcoma; RT-PCR, reverse transcriptase-PCR; NA, not available. 
Figure 3 Microscopic appearance of YWHAE-rearranged high-grade endometrial stromal sarcoma (HGESS). (A) Scanning magnification showing 'tongue-like' infiltration similar to low-grade endometrial stromal sarcoma. (B) Nested and pseudoglandular pattern $(H \& E \times 20)$. (C) Delicate linear vascular pattern and lack of perivascular whorling (H\&E $\times 20$ ). (D) Epithelioid cytomorphology with moderate amount of cytoplasm, irregular nuclear contour and nuclear clearing $(\mathrm{H} \& \mathrm{E} \times 40)$.

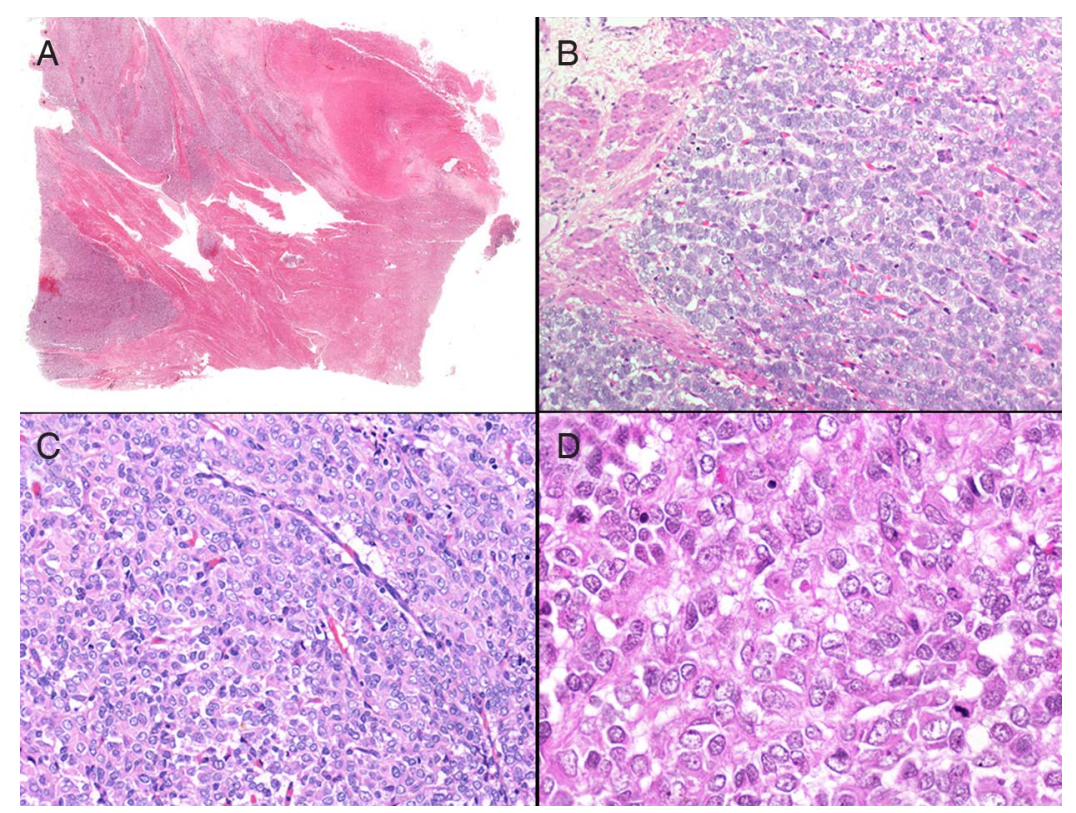

\section{Ancillary studies and differential diagnosis}

The high-grade round cell/epithelioid component of the tumour characteristically shows diffuse strong nuclear staining for cyclin $\mathrm{D} 1,{ }^{75}$ lack of CD10, and weak or absent staining for ER and PR, while the low-grade component typically shows diffuse positivity for CD10, ER and PR similar to conventional LGESS $^{66}$ (figure 4A-H). The lack of hormone receptor expression may have potential treatment implications. Diffuse cytoplasmic/membranous c-Kit expression is consistently seen in the round cell areas. ${ }^{76}$ The distinctive morphological and immunohistochemical features of HGESS are generally good surrogate markers for the underlying rearrangement, but fluorescence in situ hybridisation and/or reverse transcriptase-PCR studies may be used in equivocal cases. ${ }^{70} 77$

YWHAE-rearranged HGESS should be distinguished from LGESS because of the prognostic and treatment implications. LGESS shows smaller 'blue' cells with scanty cytoplasm and smooth nuclear contour, characteristic spiral arterioles, lack of biphasic appearance, infrequent necrosis and CD10/ER positive cyclin D1/cKit negative immunoprofile. In excision specimens, adequate sampling is necessary in order not to overlook any fibrous/fibromyxoid LGESS component that helps establish the

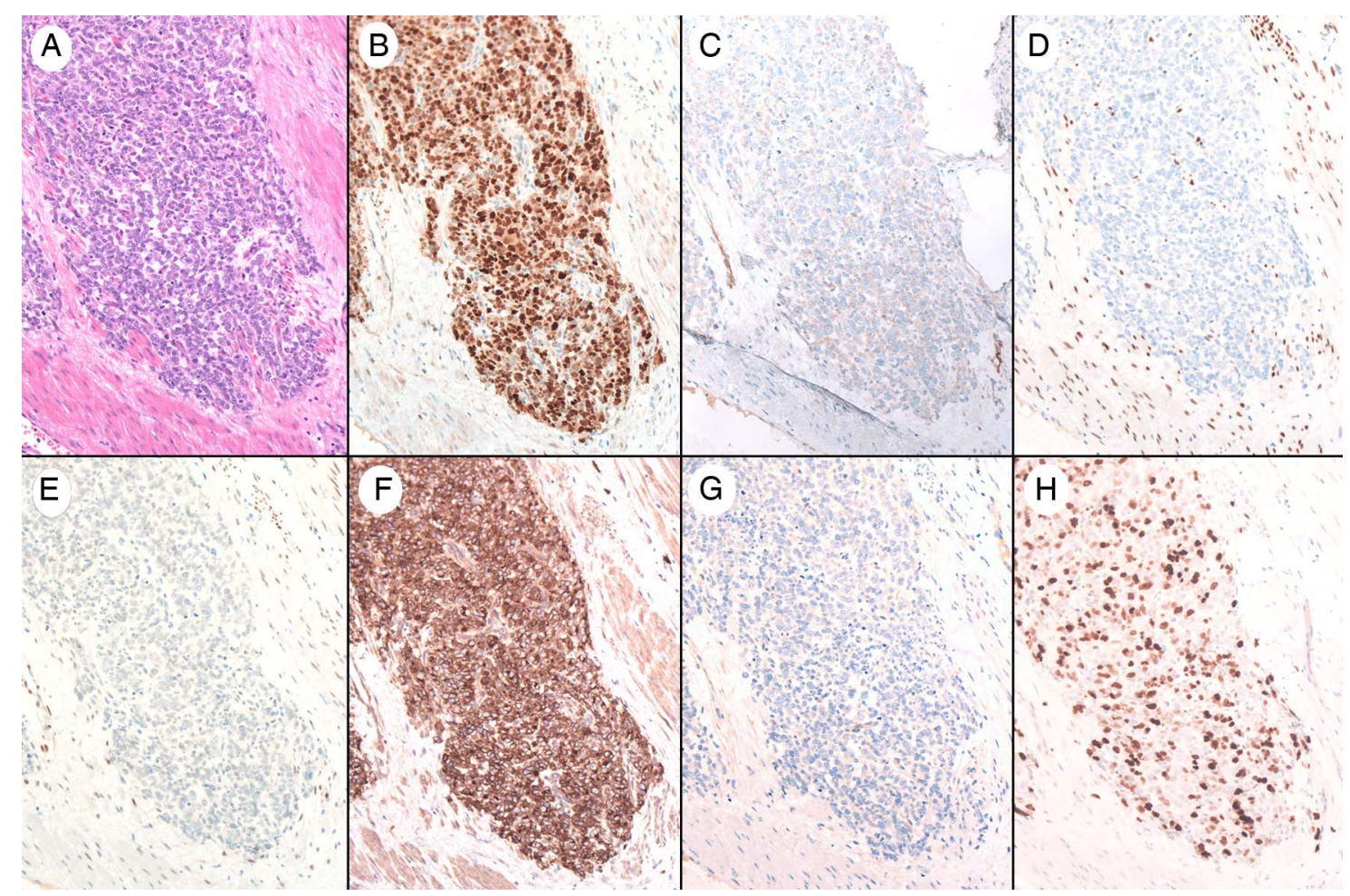

Figure 4 Immunophenotype of YWHAE-rearranged high-grade endometrial stromal sarcoma. (A) H\&E of the high-grade round cell component of the tumour. (B) Diffuse cyclin D1 nuclear immunostaining. (C) CD10 negative. (D) Oestrogen receptor negative. (E) Progesterone receptor negative. (F) Diffuse cKit staining. (G) DOG1 negative. (H) Ki67 shows a high proliferative index (20x magnification). 
diagnosis of HGESS. Epithelioid leiomyosarcoma should be excluded by appropriate smooth muscle immunomarkers, presence of at least focal marked atypia, \pm presence of conventional leiomyosarcoma. Perivascular epithelioid cell tumour (PEComa) of gynaecological origin may show 'finger-like' projections analogous to those of ESS but typically shows epithelioid and spindled cells with clear to eosinophilic cytoplasm, nested growth pattern, prominent vascular network and coexpression of melanocytic and muscle markers. ${ }^{78}$ HGESS may also be potentially confused with epithelioid gastrointestinal stromal tumour particularly in intraperitoneal/pelvic locations in view of the frequent extrauterine spread at presentation and cKit positivity. In these instances radiological-pathological correlation for possible uterine origin along with lack of DOG1 staining are often helpful. ${ }^{76}$

\section{UNDIFFERENTIATED UTERINE SARCOMA Overview}

In order to encompass tumours of endometrial and nonendometrial derivation, the term UUS has now replaced the older term 'undifferentiated endometrial sarcoma' in the updated WHO book. This wastebasket category comprises a heterogeneous group of high-grade tumours that fail to fulfil the morphological and immunohistochemical criteria of translocationpositive endometrial sarcomas. These are highly aggressive tumours, typically presenting in older patients as postmenopausal bleeding with/without manifestations of high-stage extrauterine disease. The vast majority appear to arise through genetic pathways distinct from those of LGESS and HGESS, ${ }^{79} 80$ demonstrating complex karyotypes and genomic gains and losses but lacking specific translocations. ${ }^{80} 81$ Rare cases with a coexisting LGESS component have been reported suggesting that at least a subset may arise through 'dedifferentiation' in a low-grade sarcoma. ${ }^{65} 8283$

\section{Morphological features}

Grossly, UUSs are relatively large fleshy tumours demonstrating destructive infiltrative growth into the uterine wall, typically associated with extensive necrosis and/or haemorrhage. ${ }^{84}$ Histologically, they show sheets or fascicles of highly atypical non-descript cells that do not resemble proliferative-phase endometrial stroma (figure 5). Lymphovascular invasion is common and mitotic activity is brisk and commonly associated with atypical mitotic figures.

\section{Ancillary studies and differential diagnosis}

No consistent immunophenotype is identified reflecting the heterogeneity of tumours in this category. CD10 is variably expressed $^{84}$ and can be diffuse, therefore this marker is not usually helpful in this morphological context. Hormone receptors may be negative or weakly positive.

UUS is a diagnosis of exclusion and should only be made after extensive sampling of the tumour in order to exclude other more common high-grade neoplasms with evidence of differentiation. These include leiomyosarcoma (desmin/ h-caldesmon positive, CK/EMA frequently positive), carcinosarcoma (at least focal malignant epithelial component present), rhabdomyosarcoma (rhabdomyoblasts, desmin/myogenin/ MyoD1 positive), sarcomatous overgrowth in a Mullerian adenosarcoma (at least focal low-grade areas with benign epithelial elements), undifferentiated endometrial carcinoma (uniform dyshesive cells resembling lymphocytes \pm low-grade endometrioid carcinoma component, at least focal keratin/EMA) ${ }^{85}$ and diffuse large B-cell lymphoma (resolved by appropriate immunohistochemistry).

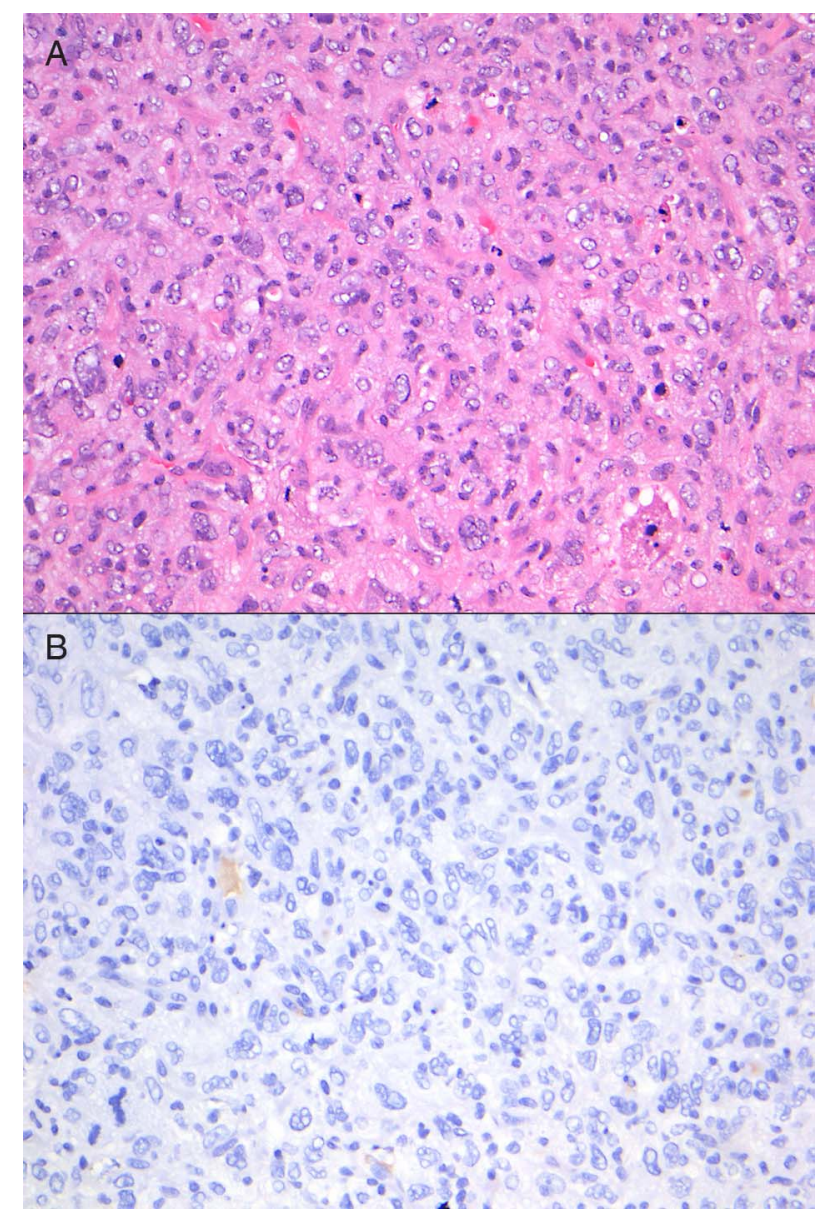

Figure 5 Undifferentiated uterine sarcoma. (A) Pleomorphic cytomorphology and brisk mitosis. (B) h-Caldesmon immunostain is negative (20x magnification).

To sum up, our understanding of ESTs has evolved dramatically in the past couple of decades despite the rarity of these tumours and overlapping morphological features. In particular, molecular advances have played a major role in characterising ESTs more objectively. Further research is required to shed more light on the underlying molecular pathways, gain more insights into pathogenesis and optimise treatment strategies as an ultimate goal.

\section{Take home messages}

- Endometrial stromal tumours are rare uterine mesenchymal neoplasms that may mimic a variety of other uterine tumours.

- The rarity of endometrial stromal tumours has made them difficult to study and classify into meaningful clinicopathological categories.

- Recent molecular advances, which are now incorporated into the 2014 World Health Organization (WHO) classification system, have objectively assisted in the classification of these tumours.

- The WHO classification system now recognizes four tumour categories with a clear distinction between low-grade and high-grade endometrial stromal sarcomas. 
Handling editor Runjan Chetty

Acknowledgements Both authors have contributed equally in the preparation of this manuscript. The authors would like to thank Dr Remaa Al-Safi for providing the photo in figure $1 \mathrm{~A}$.

Competing interests None.

Provenance and peer review Commissioned; internally peer reviewed.

\section{REFERENCES}

1 Silverberg SG, Kurman RJ, eds. Tumors of the uterine corpus and gestational trophoblastic disease. Atlas of tumor pathology. Third series, Fascicle 3. Washington DC: Armed Forces Institute of Pathology 1992:91-110.

2 Norris HJ, Taylor HB. Mesenchymal tumors of the uterus. I. A clinical and pathological study of 53 endometrial stromal tumors. Cancer 1966;19:755-66.

3 Tavassoli FA, Norris HJ. Mesenchymal tumours of the uterus. VII. A clinicopathological study of 60 endometrial stromal nodules. Histopathology 1981;5:1-10.

4 Dionigi A, Oliva E, Clement PB, et al. Endometrial stromal nodules and endometria stromal tumors with limited infiltration: a clinicopathologic study of 50 cases. Am J Surg Pathol 2002;26:567-81.

5 Lloreta J, Prat J. Ultrastructure of an endometrial stromal nodule with skeletal muscle. Ultrastruct Pathol 1993;17:405-10.

6 Lloreta J, Prat J. Endometrial stromal nodule with smooth and skeletal muscle components simulating stromal sarcoma. Int J Gynecol Pathol 1992;11:293-8.

7 Geetha V, Rupashree S, Bhat SS. Endometrial stromal nodule with smooth muscle differentiation. Indian J Pathol Microbiol 2008;51:76-7.

8 Vaideeswar $\mathrm{P}$, Madhiwale CV, Sharma JH, et al. Endometrial stromal sarcoma with sex-cord like features. Indian J Pathol Microbiol 2001;44:55-6.

9 Stemme S, Ghaderi M, Carlson JW. Diagnosis of endometrial stromal tumors: a clinicopathologic study of 25 biopsy specimens with identification of problematic areas. Am J Clin Pathol 2014;141:133-9.

10 Chiang S, Ali R, Melnyk N, et al. Frequency of known gene rearrangements in endometrial stromal tumors. Am J Surg Pathol 2011;35:1364-72.

11 Oliva E, Young RH, Clement PB, et al. Cellular benign mesenchymal tumors of the uterus. A comparative morphologic and immunohistochemical analysis of 33 highly cellular leiomyomas and six endometrial stromal nodules, two frequently confused tumors. Am J Surg Pathol 1995;19:757-68.

12 Agoff SN, Grieco VS, Garcia R, et al. Immunohistochemical distinction of endometrial stromal sarcoma and cellular leiomyoma. Appl Immunohistochem $\mathrm{Mol}$ Morphol 2001;9:164-9.

13 Rush DS, Tan J, Baergen RN, et al. h-Caldesmon, a novel smooth muscle-specific antibody, distinguishes between cellular leiomyoma and endometrial stromal sarcoma. Am J Surg Pathol 2001;25:253-8.

14 Zhu $X Q$, Shi YF, Cheng XD, et al. Immunohistochemical markers in differential diagnosis of endometrial stromal sarcoma and cellular leiomyoma. Gynecol Oncol 2004:92:71-9.

15 de Leval L, Waltregny D, Boniver J, et al. Use of histone deacetylase 8 (HDAC8), a new marker of smooth muscle differentiation, in the classification of mesenchymal tumors of the uterus. Am J Surg Pathol 2006;30:319-27.

16 Kurman RJ, Carcangiu ML, Herrington CS, et al. WHO classification of tumours of female reproductive organs. 4th edn. Volume 6. Lyon, France: IARC Press, 2014.

17 Stadsvold JL, Molpus KL, Baker JJ, et al. Conservative management of a myxoid endometrial stromal sarcoma in a 16-year-old nulliparous woman. Gynecol Oncol 2005:99:243-5.

18 Ali RH, Al-Safi R, Al-Waheeb S, et al. Molecular characterization of a population-based series of endometrial stromal sarcomas in Kuwait. Hum Pathol 2014;45:2453-62.

19 Koskas M, Morice P, Yazbeck C, et al. Conservative management of low-grade endometrial stromal sarcoma followed by pregnancy and severe recurrence. Anticancer Res 2009:29:4147-50.

20 Dong $\mathrm{R}$, Mao H, Zhang P. Conservative management of endometrial stromal sarcoma at stage III: a case report. Oncol Lett 2014;8:1234-6.

21 Oliva E, Egger JF, Young RH. Primary endometrioid stromal sarcoma of the ovary: a clinicopathologic study of 27 cases with morphologic and behavioral features similar to those of uterine low-grade endometrial stromal sarcoma. Am I Surg Pathol 2014;38:305-15.

22 Usta TA, Sonmez SE, Oztarhan A, et al. Endometrial stromal sarcoma in the abdominal wall arising from scar endometriosis. J Obstet Gynaecol 2014;34:541-2.

23 Wheelock MC, Strand CM. Endometrial sarcoma; relationship to certain instances of stromal endometriosis. Obstet Gynecol 1953;2:384-90.

24 Gerber MA, Toker C. Primary extrauterine endometrial stromal sarcoma. Arch Pathol 1970;89:477-80

25 Persaud V, Anderson MF. Endometrial stromal sarcoma of the broad ligament arising in an area of endometriosis in a paramesonephric cyst. Case report. $\mathrm{Br}$ J Obstet Gynaecol 1977;84:149-52.

26 Berkowitz RS, Ehrmann RL, Knapp RC. Endometrial stromal sarcoma arising from vaginal endometriosis. Obstet Gynecol 1978;51(1 Suppl):34s-7.
27 Morrison C, Ramirez NC, Chan JK, et al. Endometrial stromal sarcoma of the retroperitoneum. Ann Diagn Pathol 2002;6:312-18.

28 Gloor E, Schnyder P, Cikes M, et al. Endolymphatic stromal myosis. Surgical and hormonal treatment of extensive abdominal recurrence 20 years after hysterectomy. Cancer 1982;50:1888-93.

29 Bai H, Yang J, Cao D, et al. Ovary and uterus-sparing procedures for low-grade endometrial stromal sarcoma: a retrospective study of 153 cases. Gynecol Oncol 2014;132:654-60.

30 Rauh-Hain JA, del Carmen MG. Endometrial stromal sarcoma: a systematic review. Obstet Gynecol 2013;122:676-83.

31 Amant F, Floquet A, Friedlander M, et al. Gynecologic Cancer InterGroup (GCIG) consensus review for endometrial stromal sarcoma. Int J Gynecol Cancer 2014;24(9 Suppl 3):S67-72.

32 Bodner K, Bodner-Adler B, Obermair A, et al. Prognostic parameters in endometrial stromal sarcoma: a clinicopathologic study in 31 patients. Gynecol Oncol 2001;81:160-5

33 Rothmund R, Hartkopf A, Joachim C, et al. Clinical characteristics, pathological reevaluation, surgical management and adjuvant therapy of patients with endometrial stromal tumors. Arch Gynecol Obstet 2014;290:1195-200.

34 Yoon A, Park JY, Park JY, et al. Prognostic factors and outcomes in endometrial stromal sarcoma with the 2009 FIGO staging system: a multicenter review of 114 cases. Gynecol Oncol 2014;132:70-5.

35 Riopel J, Plante M, Renaud MC, et al. Lymph node metastases in low-grade endometrial stromal sarcoma. Gynecol Oncol 2005;96:402-6.

36 Amant $F$, Coosemans $A$, Debiec-Rychter $M$, et al. Clinical management of uterine sarcomas. Lancet Oncol 2009;10:1188-98.

37 Barney B, Tward JD, Skidmore T, et al. Does radiotherapy or lymphadenectomy improve survival in endometrial stromal sarcoma? Int J Gynecol Cancer 2009;19:1232-8.

38 Shah JP, Bryant CS, Kumar S, et al. Lymphadenectomy and ovarian preservation in low-grade endometrial stromal sarcoma. Obstet Gynecol 2008;112:1102-8.

39 Choi MC, Kim Gl, Hwang YY. Fertility-sparing management combined with photodynamic therapy for endometrial stromal sarcoma: a case report. Photodiagnosis Photodyn Ther 2014;11:533-6.

40 Malouf GG, Duclos J, Rey A, et al. Impact of adjuvant treatment modalities on the management of patients with stages I-II endometrial stromal sarcoma. Ann Oncol 2010;21:2102-6

41 Sampath S, Schultheiss TE, Ryu JK, et al. The role of adjuvant radiation in uterine sarcomas. Int J Radiat Oncol Biol Phys 2010;76:728-34.

42 Li N, Wu LY, Zhang HT, et al. Treatment options in stage I endometrial stromal sarcoma: a retrospective analysis of 53 cases. Gynecol Oncol 2008;108:306-11.

43 Pink $D$, Lindner $T$, Mrozek $A$, et al. Harm or benefit of hormonal treatment in metastatic low-grade endometrial stromal sarcoma: single center experience with 10 cases and review of the literature. Gynecol Oncol 2006;101:464-9.

44 Chang KL, Crabtree GS, Lim-Tan SK, et al. Primary uterine endometrial stromal neoplasms. A clinicopathologic study of 117 cases. Am J Surg Pathol 1990;14:415-38.

45 Fekete PS, Vellios F. The clinical and histologic spectrum of endometrial stromal neoplasms: a report of 41 cases. Int J Gynecol Pathol 1984;3:198-212.

46 Paulsen SM, Nielsen VT, Hansen P, et al. Endolymphatic stromal myosis with focal tubular-glandular differentiation (biphasic endometrial stromal sarcoma). Ultrastruct Pathol 1982;3:31-42.

47 Clement PB, Scully RE. Endometrial stromal sarcomas of the uterus with extensive endometrioid glandular differentiation: a report of three cases that caused problems in differential diagnosis. Int J Gynecol Pathol 1992;11:163-73.

48 Fitko R, Brainer J, Schink JC, et al. Endometrial stromal sarcoma with rhabdoid differentiation. Int J Gynecol Pathol 1990;9:379-82.

49 Oliva E, Clement PB, Young RH. Endometrial stromal tumors: an update on a group of tumors with a protean phenotype. Adv Anat Pathol 2000;7:257-81.

50 Oliva $\mathrm{E}$, Young $\mathrm{RH}$, Clement PB, et al. Myxoid and fibrous endometrial stromal tumors of the uterus: a report of 10 cases. Int J Gynecol Pathol 1999;18:310-9.

51 Yilmaz A, Rush DS, Soslow RA. Endometrial stromal sarcomas with unusual histologic features: a report of 24 primary and metastatic tumors emphasizing fibroblastic and smooth muscle differentiation. Am J Surg Pathol 2002;26:1142-50.

52 Oliva $\mathrm{E}$, Young $\mathrm{RH}$, Amin MB, et al. An immunohistochemical analysis of endometrial stromal and smooth muscle tumors of the uterus: a study of 54 cases emphasizing the importance of using a panel because of overlap in immunoreactivity for individual antibodies. Am J Surg Pathol 2002;26:403-12.

53 Nucci MR, O'Connell JT, Huettner PC, et al. h-Caldesmon expression effectively distinguishes endometrial stromal tumors from uterine smooth muscle tumors. Am J Surg Pathol 2001;25:455-63.

54 Chesnais AL, Watkin E, Beurton D, et al. Myxoid mesenchymal tumors of uterus: endometrial stromal and smooth muscle tumors, myxoid variant. Ann Pathol 2011:31:152-8.

55 Stewart CJ, Leung YC, Murch A, et al. Evaluation of fluorescence in-situ hybridization in monomorphic endometrial stromal neoplasms and their histological mimics: a review of 49 cases. Histopathology 2014;65:473-82.

56 Amador-Ortiz C, Roma AA, Huettner PC, et al. JAZF1 and JJAZ1 gene fusion in primary extrauterine endometrial stromal sarcoma. Hum Pathol 2011:42:939-46. 
57 Oliva E, de Leval L, Soslow RA, et al. High frequency of JAZF1-JJAZ1 gene fusion in endometrial stromal tumors with smooth muscle differentiation by interphase FISH detection. Am J Surg Pathol 2007;31:1277-84.

58 Nucci MR, Harburger D, Koontz J, et al. Molecular analysis of the JAZF1-JJAZ1 gene fusion by RT-PCR and fluorescence in situ hybridization in endometrial stromal neoplasms. Am J Surg Pathol 2007;31:65-70.

59 Koontz JI, Soreng AL, Nucci M, et al. Frequent fusion of the JAZF1 and JJAZ1 genes in endometrial stromal tumors. Proc Natl Acad Sci USA 2001;98:6348-53.

60 Micci F, Gorunova L, Gatius S, et al. MEAF6/PHF1 is a recurrent gene fusion in endometrial stromal sarcoma. Cancer Lett 2014;347:75-8.

61 Panagopoulos I, Micci F, Thorsen J, et al. Novel fusion of MYST/Esa1-associated factor 6 and PHF1 in endometrial stromal sarcoma. PLOS ONE 2012;7:e39354.

62 Panagopoulos I, Thorsen J, Gorunova L, et al. Fusion of the ZC3H7B and BCOR genes in endometrial stromal sarcomas carrying an X;22-translocation. Genes Chromosomes Cancer 2013;52:610-18.

63 Dewaele B, Przybyl J, Quattrone A, et al. Identification of a novel, recurrent MBTD1-CXorf67 fusion in low-grade endometrial stromal sarcoma. Int J Cancer 2014; 134:1112-22.

64 Lee $\mathrm{CH}, \mathrm{Ou}$ WB, Marino-Enriquez A, et al. 14-3-3 fusion oncogenes in high-grade endometrial stromal sarcoma. Proc Natl Acad Sci USA 2012;109:929-34.

65 Kurihara S, Oda Y, Ohishi Y, et al. Endometrial stromal sarcomas and related high-grade sarcomas: immunohistochemical and molecular genetic study of 31 cases. Am J Surg Pathol 2008;32:1228-38.

66 Lee $\mathrm{CH}$, Marino-Enriquez A, Ou W, et al. The clinicopathologic features of YWHAE-FAM22 endometrial stromal sarcomas: a histologically high-grade and clinically aggressive tumor. Am J Surg Pathol 2012;36:641-53.

67 Kruse AJ, Croce $S$, Kruitwagen RF, et al. Aggressive behavior and poor prognosis of endometrial stromal sarcomas with YWHAE-FAM22 rearrangement indicate the clinical importance to recognize this subset. Int J Gynecol Cancer 2014;24:1616-22.

68 Gremel G, Liew M, Hamzei F, et al. A prognosis based classification of undifferentiated uterine sarcomas: identification of mitotic index, hormone receptors and YWHAE-FAM22 translocation status as predictors of survival. Int I Cancer 2014. Published Online First: 14 Aug 2014.

69 Sciallis AP, Bedroske PP, Schoolmeester JK, et al. High-grade endometrial stromal sarcomas: a clinicopathologic study of a group of tumors with heterogenous morphologic and genetic features. Am J Surg Pathol 2014;38:1161-72.

70 Croce S, Hostein I, Ribeiro A, et al. YWHAE rearrangement identified by FISH and RT-PCR in endometrial stromal sarcomas: genetic and pathological correlations. Mod Pathol 2013:26:1390-400.

71 Amant F, Tousseyn T, Coenegrachts $L$, et al. Case report of a poorly differentiated uterine tumour with $\mathrm{t}(10 ; 17)$ translocation and neuroectodermal phenotype. Anticancer Res 2011;31:2367-71.
72 Regauer S, Emberger W, Reich 0 , et al. Cytogenetic analyses of two new cases of endometrial stromal sarcoma-non-random reciprocal translocation $t(10 ; 17)$ (q22;p13) correlates with fibrous ESS. Histopathology 2008;52:780-3.

73 Micci F, Walter CU, Teixeira MR, et al. Cytogenetic and molecular genetic analyses of endometrial stromal sarcoma: nonrandom involvement of chromosome arms $6 p$ and $7 p$ and confirmation of JAZF1/JJAZ1 gene fusion in $t(7 ; 17)$. Cancer Genet Cytogenet 2003;144:119-24.

74 Leunen K, Amant F, Debiec-Rychter M, et al. Endometrial stromal sarcoma presenting as postpartum haemorrhage: report of a case with a sole $t(10 ; 17)$ (q22;p13) translocation. Gynecol Oncol 2003;91:265-71.

75 Lee $\mathrm{CH}$, Ali RH, Rouzbahman M, et al. Cyclin D1 as a diagnostic immunomarker for endometrial stromal sarcoma with YWHAE-FAM22 rearrangement. Am J Surg Pathol 2012:36:1562-70.

76 Lee $\mathrm{CH}$, Hoang LN, Yip S, et al. Frequent expression of KIT in endometrial stromal sarcoma with YWHAE genetic rearrangement. Mod Pathol 2014; 27:751-7.

77 Isphording A, Ali RH, Irving J, et al. YWHAE-FAM22 endometrial stromal sarcoma: diagnosis by reverse transcription-polymerase chain reaction in formalin-fixed, paraffin-embedded tumor. Hum Pathol 2013;44:837-43.

78 Schoolmeester JK, Howitt BE, Hirsch MS, et al. Perivascular epithelioid cell neoplasm (PEComa) of the gynecologic tract: clinicopathologic and immunohistochemical characterization of 16 cases. Am J Surg Pathol 2014;38:176-88

79 Chiang S, Oliva E. Cytogenetic and molecular aberrations in endometrial stromal tumors. Hum Pathol 2011;42:609-17.

80 Halbwedl I, Ullmann R, Kremser ML, et al. Chromosomal alterations in low-grade endometrial stromal sarcoma and undifferentiated endometrial sarcoma as detected by comparative genomic hybridization. Gynecol Oncol 2005;97: 582-7.

81 Gil-Benso R, Lopez-Gines C, Navarro S, et al. Endometrial stromal sarcomas: immunohistochemical, electron microscopical and cytogenetic findings in two cases. Virchows Archiv 1999;434:307-14.

82 Amant $F$, Woestenborghs $H$, Vandenbroucke $V$, et al. Transition of endometrial stromal sarcoma into high-grade sarcoma. Gynecol Oncol 2006;103:1137-40.

83 Ohta $Y$, Suzuki T, Omatsu $M$, et al. Transition from low-grade endometrial stromal sarcoma to high-grade endometrial stromal sarcoma. Int J Gynecol Pathol 2010;29:374-7.

84 Oliva E. Cellular mesenchymal tumors of the uterus: a review emphasizing recent observations. Int J Gynecol Pathol 2014;33:374-84.

85 Tafe LJ, Garg K, Chew I, et al. Endometrial and ovarian carcinomas with undifferentiated components: clinically aggressive and frequently underrecognized neoplasms. Mod Pathol 2010;23:781-9. 